\title{
Think Tanks, saber experto y formación de agenda política en el Chile actual*
}

\author{
Juan Pablo Pinilla \\ Universidad de Chile, Santiago, Chile. Email: jppinilla@ug.uchile.cl
}

\begin{abstract}
Resumen: El artículo indaga en los canales de influencia de los centros de estudio privados o think tanks en el proceso de formación de agenda política en Chile. Un esquema de clasificación para las organizaciones existentes en el país es propuesto, caracterizando los rasgos de centros académicos, centros de apoyo, centros partidarios y centros de gestión. Se busca describir el desenvolvimiento de las distintas organizaciones y determinar el rol de la gestión de saber experto en la definición de asuntos para la agenda política.

Palabras clave: Think Tanks; Políticas de la Experticia; Saber Experto; Agenda Política.
\end{abstract}

\section{Think tanks, expert knowledge and political agenda setting in Chile today}

\begin{abstract}
The paper explores the channels of influence of think tanks in the process of political agenda setting in Chile. A classification scheme for organizations in the country is proposed, characterizing the features of academic think tanks, advocacy think tanks, party think tanks and contract think tanks. It seeks to describe the performance of different types of organizations, and determine the role of knowledge expert management in defining issues of the political agenda.
\end{abstract} Agenda.

Key words: Think Tanks; Politics of Expertise; Expert Knowledge; Political

\section{Think tanks, conhecimento especializado e definição da agenda política no Chile hoje}

Resumo: O artigo explora os canais de influência dos think tanks no processo de definição de agenda política no Chile. Um esquema de classificação para as organizações no país é proposto, caracterizando as características de grupos de reflexão acadêmica, a defesa think tanks, grupos de reflexão e festa contrato think tanks. Pretende-se descrever o desempenho de diferentes tipos de organizações, e determinar o papel de especialista em gestão do conhecimento na definição de temas da agenda política.

Palavras-chave: Think tanks; Política de especialização; conhecimento perito; agenda política. 


\section{Introducción}

En un mundo cada vez más interconectado, las políticas públicas, sean originadas en gobiernos, por influencia de grupos empresariales, entidades supranacionales, organizaciones no gubernamentales, actores privados o alguna combinación entre estos, representan un medio de intervención cada vez más relevante en la sociedad (Wedel et. al., 2005: 31). Actualmente la formulación de políticas congrega a una red heterogénea de actores más o menos dispersos, vinculados entre sí por relaciones de poder, recursos, ideologías e influencias interdependientes. Es en esta articulación donde el Estado ya no se relaciona a una esfera pública amorfa, sino, y cada vez con más frecuencia, con "actores corporativos que disponen de sus propias bases de poder” (Willke, 2006: 185). Los distintos colectivos de la sociedad civil buscan hacerse partícipes del proceso político y jugar un rol importante -aunque a menudo difuso e indirecto- en la configuración de los asuntos públicos. En este marco han ganado visibilidad nuevos actores privados. Tales referentes tienden a circular por fuera de las instituciones académicas tradicionales, formando organizacionales de diverso giro, entre las que se hallan firmas consultoras, entidades internacionales, organizaciones no gubernamentales, y centros de estudio privados o think tanks.

Con un incremento sostenido durante las últimas cuatro décadas, los así denominados think tanks se encuentran hoy entre las más activas instituciones expertas con interés por la política pública; especialmente en países como Estados Unidos, Canadá y Gran Bretaña, donde representan agentes gravitantes del escenario político (Abelson, 2009; Rich, 2004; Abelson \& Carberry, 1998). Abocados de manera general al ejercicio de asesoramiento e influencia, su presencia se ha multiplicado alcanzando unos seis mil cuatrocientos centros repartidos por 120 países (McGann, 2011). Una de las razones que explican su protagonismo se asocia a la abundancia creciente de informaciones que deben procesar los tomadores de decisiones, frente a lo cual se vuelve imprescindible contar con referentes donde obtener consejos oportunos y análisis de controversias públicas (Limoges, 1993). Frente a esto, el recurso al saber experto se ha tornado recurrente en las distintas fases del proceso político en regímenes democráticos (Fisher, 2009).

En los intersticios del vínculo entre Estado, partidos políticos y sociedad civil, los think tanks se han destacado como detentadores de determinada expertise. Uno de los ámbitos en los cuales parecen desempeñarse con relativo éxito dice relación con la tematización y visibilización de temas públicos. ¿Cómo los think tanks locales participan de la definición de agendas? ¿Es posible hallar particularidades en las intervenciones de las distintas organizaciones? ¿Qué lugar tiene la expertise gestionada en los centros y cómo sus expertos logran obtener mejores oportunidades para colocar temas en el debate político? Son algunas de las cuestiones que guían el texto.

Para abordar estos problemas se introduce en primer lugar la noción de "políticas de la experticia”, con objeto de aludir a los nuevos condicionamientos que modelan la formulación de políticas en la actuali- 
dad. Luego se hace referencia a los think tanks, en tanto organizaciones especializadas en la gestión de conocimiento experto. Haciendo uso de una tipología que ordena los centros de estudio privados locales según sus orientaciones académicas (academic think tanks), de apoyo (advocacy think tanks), de gestión (contract think tanks) y partidarias (party think tanks); se analiza el desenvolvimiento de los think tanks en la construcción de agenda política, identificando sus medios de intervención e influencia. El artículo cierra con una reflexión sobre el proceder de los centros de estudio y la gestión de la experticia en el horizonte de la participación ciudadana y la calidad de la democracia en Chile.

\section{Políticas de la experticia y think tanks}

En la actualidad es posible constatar que la premisa "normativa" que guía la toma de decisiones democrática comparte espacio con premisas de tipo "cognitivas", sustentadas en la creencia de que las decisiones de los asuntos públicos pueden basarse en conocimientos y consideraciones de experticia (Willke, 2007: 35-6). Los recursos que ofrece la ciencia aplicada se incorporan la formulación de políticas, dando lugar a espacios para que una experticia individual o colectiva tome parte en la deliberación de la política pública.

La provisión de evidencia oportuna para la toma de decisiones adquiere entonces relevancia crítica (European Commission, 2008; UNESCO \& ISSC, 2010; Duflo \& Takavarasha, 2010). Las investigaciones técnicas, se asegura, brindan lineamientos que pueden llegar a permear y transformar los modos de pensar los temas de política, tanto a nivel de la opinión pública como de las autoridades electas (Rich, 2004). Las “ideas” pueden incentivar la acción proveyendo "hojas de ruta” particulares en los dilemas de política; empujando a los encargados de la toma de decisiones a seguir direcciones precisas y brindándoles buenas razones para adoptar determinados cursos de acción (Campbell, 1998). Para esto el recurso a la experticia resulta fundamental, en la medida que permite dotar de credibilidad técnica un saber que puede servir de base para la deliberación entre las autoridades.

En el marco de una gradual extensión de políticas basadas en experticia, la "gestión de saber experto” sirve como vía de traducción entre evidencia científica y decisión política al momento de evaluar alternativas (Pinilla y Godoy, 2010). Bajo estas nociones, la idea de políticas basadas en experticia alude a un marco para la discusión de los asuntos públicos caracterizado por (a) la aparición de organizaciones público-privadas con interés por la definición de lo público; (b) la incorporación de un "actor experto” al juego político; y (c) la emergencia de un medio simbólico denominado "experticia".

En el contexto latinoamericano el auge de las políticas de la experticia se ha manifestado en un creciente número de expertos, en general ligados al área económica y financiera, que pasan a ocupar posiciones gravitantes 
en el sector público y privado, especialmente desde la década del sesenta en adelante (Centeno \& Silva, 1998; Markoff \& Montecinos, 1994; Centeno, 1993). Es así como se expande un nuevo recurso para otorgar validez y razonabilidad técnica a la formulación de políticas. Cabe indicar, en esta dirección, que la "experticia” no se funda en un conocimiento neutro en términos normativos o valóricos: su vara de medición está lejos de los estándares de producción científica de la academia. Todavía más, cuando un actor social o una organización ofrecen su experticia en una materia determinada, el valor de ésta no radica en atributos de verdad o falsedad. Como indican autores,

"no se trata de un conocimiento teórico propio de la academia y de las universidades, sino de un tipo de subproducto experto dirigido a quienes toman decisiones y cuyo objetivo es su aplicabilidad en el terreno práctico” (Gárate, 2008: 68).

En efecto, existe cierto acuerdo en señalar que la experticia no funda su efectividad en la cualidad de verdad del saber (Fischer, 2009: 143), sino más bien en su aplicabilidad en servicio de ciertas decisiones o acciones (Limoges, 1993). La experticia puede comprenderse, en consecuencia, como una forma elaborada de la influencia social, que ha conseguido cierta especialización al apoyarse en un marco institucional y en organizaciones particulares, una de las cuales son los think tanks.

Los think tanks han llegado a representar, en efecto, las organizaciones más numerosas dedicadas a la investigación y asesoría en políticas (Abelson, 2009; Rich, 2004). A menudo se los ha considerados como centros de pensamiento cuyo fuerte reside en la especialización temática y la expertise que desarrollan en ámbitos de política particulares (McGann, 2008). Se caracterizan por ofrecer orientaciones y consejos técnicos a partidos políticos y gobiernos de turno, así como por establecer vínculos con variados actores, entre ellos universidades, grupos de interés, fundaciones, organizaciones no gubernamentales, sindicatos, y gremios empresariales.

Con independencia de los éntasis puestos por los autores (Cf. Rich, 2004: 11; Bellettini, 2007: 113; Uña, 2007: 180), los think tanks pueden definirse como organizaciones privadas -centros, institutos, fundaciones-, que, manteniendo cierta autonomía, aspiran a la intervención en políticas públicas y la influencia en la toma de decisiones por medio de la producción de ideas y la gestión de experticias (Pinilla y Godoy, 2010). En América Latina organizaciones de estas características no aparecen sino hasta inicios de los años sesenta. Pero es durante los procesos de transición democrática cuando los think tanks y centros de estudios comienzan a tener protagonismo en la región (Brunner, 1985; Puryear, 1994; Gárate, 2008). Durante la década de 1990 se asiste a lo que podemos denominar una tercera generación de centros. El retorno a la democracia permite la difusión de actores privados, entre ellos think tanks y expertos vinculados a las reformas económicas y al diseño de un nuevo marco institucional (Belletini, 2007: 114). Las investigaciones sobre la experiencia chilena han puesto 
especial atención en los casos de la política económica, subrayando la presencia de los denominados Chicago Boys, los Monjes del CIEPLAN y otros expertos que participaron en instituciones privadas y luego se desempeñaron activamente durante los Gobierno de la Concertación (Gárate, 2010; Silva, 2006, 1991).

Valiéndonos de un criterio de operacional, se ha aplicado una clasificación que permite visualizar las diferencias entre los think tanks chilenos; considerando que estas organizaciones han debido adaptar sus medios y canales de influencia a distintos ámbitos, especializando funciones y definiendo prioridades. El esquema busca identificar funciones prioritarias, sacando a la luz los rasgos más distintivos de cada organización. ${ }^{1}$

\section{Figura 1. Esquema de clasificación de think tanks en Chile}

\begin{tabular}{|c|c|}
\hline & Organizaciones \\
\hline $\begin{array}{l}\text { Centros de Apoyo } \\
\text { (Advocacy) }\end{array}$ & $\begin{array}{l}\text { Centro de Estudios de la Realidad Contemporánea CERC; Fundación Salvador } \\
\text { Allende; Fundación Democracia y Desarrollo FDD; Fundación Frei; Instituto } \\
\text { Democracia y Mercado IDM; Libertad y Desarrollo LyD; Corporación de } \\
\text { Estudios Sociales y Educación SUR; Fundación Chile 21; Proyectamérica }\end{array}$ \\
\hline $\begin{array}{l}\text { Centros } \\
\text { Partidarios (Partv) }\end{array}$ & $\begin{array}{l}\text { Fundación Jaime Guzmán FJG; Instituto Igualdad; Instituto Libertad; Instituto } \\
\text { de Ciencias Alejandro Lipschutz ICAL; Centro de Estudios Sociales } \\
\text { AVANCE }\end{array}$ \\
\hline $\begin{array}{l}\text { Centros de } \\
\text { Gestión (Contract) }\end{array}$ & Fundación Paz Ciudadana FPC \\
\hline $\begin{array}{l}\text { Centros } \\
\text { Académicos } \\
\text { (Academic) }\end{array}$ & $\begin{array}{l}\text { Centro de Estudios Públicos CEP; Centro de Estudios del Desarrollo CED; } \\
\text { Centro de Estudios Nacionales de Desarrollo Alternativo CENDA; } \\
\text { Corporación de Promoción Universitaria CPU; Centros de Estudios de la } \\
\text { Mujer CEM; Corporación EXPANSIVA; Corporación de Estudios para } \\
\text { Latinoamérica CIEPLAN }\end{array}$ \\
\hline
\end{tabular}

Fuente: Pinilla y Godoy (2010). Datos en sitios web y entrevistas de los autores.

El grueso de organizaciones registradas asume la forma de "centros de apoyo" o advocacy think tanks; esto es, instituciones que otorgan un respaldo experto a la promoción de ideas y valores de los distintos actores involucrados en la definición de los asuntos públicos. Los "centros partidarios" o party think tanks pueden considerarse una variación de los anteriores, sólo que aquí el vínculo con un partido político específico es directo y generalmente explícito. En centros de gestión o contract think tanks sólo califica una organización, en parte por sus similitudes con oficinas de consultoría y de asesorías, que carecen de pretensiones de incidencia sobre la esfera pública y poseen más bien fines de lucro. Finalmente, los academic 
think tanks o también llamados "universidades sin estudiantes” están marcados por un desarrollo en el área de investigación y publicación (propias o de terceros). Se caracterizan por una fuerte confianza en académicos e investigadores, financiados principalmente por el sector privado, y estudios en detalle como principal producto.

Los distintos centros han colaborado a la formación de un diálogo político, generando alternativas y opciones para los tomadores de decisiones. Sin embargo, las intervenciones se realizan de diferentes maneras y en distintas etapas del ciclo de políticas. A continuación se expondrán los medios de influencia característicos de las distintas organizaciones, poniendo el foco en el proceso de formación de agenda y priorización de asuntos para la política.

\section{Think tanks y formación de agenda política}

\section{Think tanks y gestión de la experticia}

¿A qué se debe que algunos asuntos lleguen a formar parte de las prioridades de los gobiernos, mientras que otros quedan olvidados o son pasados por alto? Esta tal vez sea una de las principales interrogantes del estudio del ciclo de políticas públicas, y uno de los aspectos que cualquier analista debe tener en consideración hoy (Howlett \& Ramesh, 2003). El cómo emergen y llegan a definirse los asuntos públicos tiene consecuencias a la hora de proponer alternativas y formular políticas. Como han destacado los investigadores, la temprana definición de los temas, su visibilización y problematización, dice mucho respecto a los modos de resolución pertinentes (Kingdon, 1995; Rich, 2004).

El concepto de "agenda" puede ser comprendido como el listado de temas y problemas a los cuales oficiales de gobierno, y agentes extragubernamentales fuertemente vinculados a ellos, se encuentran prestando atención en un momento dado (Kingdon, 1995: 3). De este modo, la formación de agendas propone llamar la atención sobre el proceso dinámico de visibilización y reconocimiento de temas para la política (Howlett \& Ramesh, 2003: 120ss). El interés estriba entonces en especificar el rol de los think tanks locales en el establecimiento de la agenda política, comparando el desempeño y particularidades de cada tipo de organización.

Debe considerarse que los centros tienen un interés creado en dar la impresión de que ejercen una influencia importante, por lo que es fácil sobreestimar el éxito de sus ofertas de experticia (Abelson, 2007). Ante esto, resulta razonable partir del supuesto que los think tanks poseen diferentes grados de influencia, y que utilizan canales específicos para hacer valer sus intereses y “fijar la agenda” (Braun et. al., 2007).

En el caso de los centros de apoyo, sus actividades se encuentran estrechamente ligadas a la prestación de experticia a programas políticos 
amplios. El vínculo ideológico que sostienen con grupos de interés, sumado a su dependencia de fuentes de financiamiento, hace que uno de los desafíos de los centros sea garantizar una autonomía suficiente, de modo tal que su imagen pública no se vea afectada de manera permanente. En efecto, muchas de estas organizaciones reciben recursos de entidades internacionales como la Fundación Hanns Seidel, de orientación socialcristiana conservadora; la Fundación Friedrich Ebert, de cariz socialdemócrata y ligada al Partido Socialista; la Fundación Konrad-Adenauer, de corte socialcristiano vinculado a la Democracia Cristiana; o la Fundación Rosa Luxemburgo, ligada al Partido Comunista.

En el país estos centros tradicionalmente se alinean a conglomerados políticos. Libertad y Desarrollo ha sido, por ejemplo, el principal referente en la prestación de experticia técnica y suministro de ideas a la Alianza por Chile y la Coalición por el Cambio. Uno de sus fundadores, Cristián Larroulet, pasó a formar parte del Gobierno de Sebastián Piñera en calidad de Ministro Secretario General de la Presidencia. Representando uno de los think tanks más importantes de Chile y América Latina, hoy forma parte, junto con el Instituto Democracia y Mercado, de la Red Liberal de América Latina (RELIAL), una plataforma para fortalecer la cooperación y colaboración de instituciones de la región de corte liberal.

Otra organización importante corresponde a la Corporación Expansiva, organización fundada por el ex Ministro de Hacienda Andrés Velasco. En el centro confluyeron sectores socialdemócratas y liberales, y se caracterizó por tener una importante injerencia en el Gobierno de Michelle Bachelet (Silva, 2007). Luego, bajo el Directorio de Jorge Marshall, la organización se vinculó al Instituto de Políticas Públicas de la Universidad Diego Portales, refundando Expansiva-UDP. Esta configuración, única en el país, hizo de Expansiva un centro con carácter de difusión académica hasta mediados de 2010, cuando la alianza se rompió debido al interés de la universidad por acentuar el carácter académico de sus programas.

CIEPLAN representa uno de los centros de apoyo de mayor gravitación en la historia del Chile actual. Fundada por figuras de la política nacional tales como Alejandro Foxley, José Pablo Arellano y René Cortázar, fue históricamente asociado a la gestión tecnócrata de la experticia sobre políticas económicas durante la transición democrática (Gárate, 2010; Silva, 1991). Hoy en día abocado a instalar y difundir una agenda de crecimiento y equidad para Latinoamérica, CIEPLAN sigue concertando a importantes asesores y expertos del espectro político, entre ellos Eugenio Tironi, Patricio Meller y Francisco Javier Díaz.

Un aspecto clave en la gestión de experticia de estas organizaciones dice relación con el manejo mediático y la construcción de una imagen pública. Con este propósito, los centros se han especializado tanto en la difusión de sus productos como en la intervención en medios de comunicación. Dentro de lo primero, se pone énfasis en la simplificación del conoci- 
miento experto y su difusión a través de diversas series de publicaciones. Como indicó uno de los consultados,

“el trabajo científico o académico también debe tener una dimensión de vulgarización, en circunstancias en que hay que saber masificar un conocimiento complejo” (Alfredo Joignant, Corporación Expansiva).

De este modo, publicaciones como Estudios y Propuestas de CIEPLAN, Serie en Foco de Expansiva, Temas Públicos de Libertad y Desarrollo, Colección Ideas de Chile 21, han logrado llegar a una audiencia heterogénea, formada por asesores, decisores políticos, parlamentarios, investigadores y expertos de entidades internacionales.

En lo relativo a la exposición mediática, los centros de apoyo han desplegado esfuerzos para que sus académicos aparezcan con frecuencia en periódicos y portales Web. Los expertos expresan opiniones institucionales e individuales sobre un amplio espectro de temas de política coyuntural y de mediano plazo. Ejemplo de ello es la sección de columnas de opinión que La Segunda ha dedicado a expositores de think tanks nacionales, así como columnistas estables en medios como El Mercurio, La Tercera y El Mostrador.

La aproximación a los temas de agenda en tales centros emana desde preceptos normativos generales, como son los contenidos en las declaraciones de principios de las organizaciones. Comúnmente aluden a pautas valóricas que decantan en lineamientos para inspirar la labor del think tank: gremialistas, social demócratas, libertarios, liberal cristianos, etc. En ciertos casos remiten a la figura de un actor político de renombre, y en otros a grupos de interés que apoyan el centro vía transferencia de recursos -bajo la figura de donaciones corporativas o desde personas naturales-. La expresión de esta matriz ideológica permea las investigaciones, así como sus intervenciones en la agenda.

Los centros suelen operar además como sedes de reunión entre figuras prominentes de la política local e internacional. Brindar esta plataforma les otorga acceso a encargados de la toma de decisiones, permitiendo presentar sus ideas y garantizar una recepción positiva de las recomendaciones. El énfasis recae sobre los contenidos de la agenda gubernamental y parlamentaria, donde los asuntos reciben un tratamiento técnico por parte de los think tanks, bajo la forma de análisis de coyuntura política, comentarios y propuestas específicas.

Los centros partidarios, por su parte, representan una especialización de las formas organizacionales anteriores. Su aparición se vincula a requerimientos de gestión de saber experto para apoyar a los representantes de partidos políticos, y a la profesionalización del ejercicio de la política en general (Silva, 2006; Joignant, 2004). En efecto, los party think tanks se configuran cubriendo el espectro ideológico y partidario del país. Sus 
esfuerzos se canalizan en la síntesis y reelaboración de asuntos de política, más que llevar a cabo investigaciones originales. Dado que existe la idea de un proyecto político compartido, los think tanks deben poder generar una identidad propia, conservando su autonomía y carácter pese a la relación que mantienen con un partido político en concreto. Con este fin se han adoptado estrategias para generar una imagen pública propia, así como esfuerzos para la consecución de financiamiento y un patrimonio independiente.

El partido provee a los think tanks de acceso a parlamentarios y miembros de Gobierno a nivel central y local. En este sentido, la gestión de su experticia recorre redes que tienen menos relación con la opinión pública y una mayor concentración en autoridades políticas. Especialmente relevante es llegar a los formuladores de política, a los cuales el centro ofrece una asesoría confiable y puntual sobre asuntos de la coyuntura. En conjunto con los centros de apoyo, los think tanks partidarios han trabajado de cerca en la agenda parlamentaria. Con este objeto organizaciones como el Instituto Liberad, la Fundación Jaime Guzmán y Libertad y Desarrollo participan en la asesoría a parlamentarios de coaliciones políticas afines.

Ahora bien, el rasgo distintivo de los centros partidarios se juega en una prestación proactiva, asociada a intervenciones y trabajo de formación con jóvenes. Como sostiene un informante,

“nosotros, a diferencia de otros centros de estudio 'puros' por decirlo así, no somos un tradicional think tank, sino que somos un think 'and do' tank. Somos un think tank que tiene esta otra pata en el do, algo ‘ejecutivo’ por llamarlo así’ (Jaime Bellolio, Fundación Jaime Guzmán).

Los medios para influir en la agenda política no se limitan entonces a la provisión de ideas y recomendaciones, sino que además existe una colaboración práctica en candidaturas, campañas electorales, enrolamiento de personas para cargos en el Gobierno, entre otras actividades. Puede sostenerse, en este sentido, que los think tanks partidarios gozan de capacidades instaladas para la generación y renovación de capital político. Centros de distinto color organizan periódicamente talleres, cursos de verano, seminarios y actividades de extensión para difundir concepciones políticas. Ejemplo de ello es Jóvenes al Servicio de Chile, un programa de la Fundación Jaime Guzmán destinado a jóvenes que deseen trabajar voluntariamente en municipalidades e instituciones públicas, y el Bachillerato en Servicio Público, curso complementario a la formación universitaria basado en los ideales de la fundación.

En síntesis, la labor de estos think tanks se ha especializado en el establecimiento de vínculos de co-dependencia con partidos políticos (Baier \& Bakvis, 2007). La gestión de la experticia se halla aquí ligada a la formación de capital político y a acciones que vinculan líderes universitarios, dirigentes sociales y sindicales. En esta tarea, ICAL, Insti- 
tuto Libertad, Avance y Fundación Jaime Guzmán han logrado una importante experiencia.

Los centros de gestión guardan fuertes similitudes con organizaciones que, bajo la oferta de saber técnico, trabajan en asesorías de imagen, comunicación y consultoría a organismos públicos y privados. Tal es el caso de firmas como Imaginaccion Consultores y Asesorías para el Desarrollo. Sin embargo, en el caso de los contract think tanks existen dos rasgos que permiten su discriminación: el interés por los asuntos públicos más allá de lo que la contraparte pueda solicitar, y su carácter no orientado al lucro. Son estas dos características las que hacen de Fundación Paz Ciudadana un centro de gestión.

Si bien la organización surge en un comienzo como un centro de apoyo, con un fuerte sesgo ideológico imprimido por la elite empresarial principalmente por su presidente y fundador, Agustín Edwards Eastman-, a mediados de los noventa comienza a adquirir mayor interés por la política pública desde un punto de vista técnico. Su labor recae hoy sobre la investigación, evaluación y asesoría en materia de políticas de prevención del delito y la criminalidad. Como declara un informante:

“el lugar que ocupa la fundación es el de un ente de una trayectoria tal que apunta a contribuir, independientemente del color político de la autoridad en el Gobierno, técnicamente con conocimiento e información. Y estoy usando dos palabras que no son azarosas para nosotros: conocimiento e información. Información: encuestas, datos. Pero también conocimiento, que tiene que ver con aquello que se ha aprendido y que contribuye a hacer mejor las cosas” (Patricio Tudela, Fundación Paz Ciudadana).

Los centros de gestión se caracterizan por respaldar su trabajo en estudios técnicos, muchos de ellos especializados, con lo cual evalúan intervenciones gubernamentales y realizan sugerencias de diseño a un nivel de especificidad que otros centros difícilmente pueden alcanzar. Las actividades de las organizaciones por lo general se coordinan con agencias de gobierno especializadas en ciertas áreas de política. En el caso citado, a través de colaboraciones con el Ministerio del Interior, el Ministerio de Justicia y el SENAME.

Los denominados centros académicos, por último, se distinguen por un desenvolvimiento público fuerte, que conjuga preceptos ideológicos débiles con elementos de producción e investigación similares al trabajo de centros de estudio universitarios. No obstante, a diferencia de estos últimos, los think tanks no poseen restricciones disciplinarias ni de acceso a actores políticos. Por otro lado, si bien manifiestan lineamientos ideológicos, las normas profesionales que rigen la gestión de su experticia suelen ser mayores en relación a los centros de apoyo. Al igual que los centros de gestión, los academic think tanks desarrollan cierta especialización temática. Tal es el caso, por ejemplo, del CEM y las investigaciones aplicadas en género; el CENDA y los temas de coyuntura económica y laboral; y la CPU 
para el área de educación. No obstante, tanto la cobertura como las perspectivas para abordar sus campos de interés suelen ser más amplias y transversales que el filtro técnico de los centros anteriores.

Tal vez el rasgo que mejor representa a los centros académicos sea su capacidad para instalar agendas de trabajo propias, con extensiones de mediano y largo plazo. Esto representa un logro importante, si se considera que los tiempos de la política pública y el debate político suelen fundarse en sucesivas coyunturas. La extensión temporal de la labor de los centros académicos les ha dotado de continuidad en sus propuestas, además de investigadores con dedicación completa y gran experiencia en los temas de su experticia. Como afirma una voz experta,

"si tú revisas nuestras revistas y nuestras publicaciones en general no son tan coyunturales. El mayor evento coyuntural es la encuesta CEP. Pero desde el punto de vista de nuestra investigación, salvo excepciones, cuando un investigador tiene ventajas comparativas en un área específica, esta es pensada en el mediano y largo plazo" (Harald Beyer, Centro de Estudios Públicos).

Con independencia de los énfasis de cada organización, los centros académicos invierten ingentes recursos en la realización de las actividades de extensión y difusión. Suelen ser anfitriones recurrentes de foros, debates y seminarios, especialmente para audiencias académicas, investigadores en formación y estudiantes universitarios.

En Chile, los centros académicos han encontrado nuevas oportunidades para influir en los temas de la agenda política desde la apertura de comisiones tanto a nivel presidencial como ministerial. En efecto, estas instancias han dado espacio a la inclusión de expertos de diverso signo en la discusión de problemas públicos (Aguilera, 2009, 2007), entre ellos a miembros de equipos directivos e investigadores asociados a los think tanks locales. Uno de los casos mejor estudiados corresponde a los Consejos Asesores Presidenciales, convocados durante la presidencia de M. Bachelet para el estudio y construcción de propuestas de política en ámbitos clave de la agenda país. Su composición cuenta con la presencia de figuras de renombre de los principales centros académicos y de apoyo; donde los think tanks intervienen incorporando a sus expertos, aportando con informes y artículos de los puntos en debate, delineando propuestas y participando en las audiencias.

\section{Think tanks, sus vías de influencia y principales productos}

Un ambiente altamente competitivo, demandas de experticia y provisión de recursos, incentiva a los think tanks locales a desarrollar estrategias para hacer valer sus intereses en las distintas instancias del proceso de políticas, garantizando la gestión exitosa de su experticia. Cabe constatar que existen particularidades en los medios de intervención utilizados por los distintos tipos de centros. Variaciones que, desde luego, van asociadas 
al tamaño y disponibilidad de recursos financieros y humanos; aunque desde un punto de vista operativo, el esquema de clasificación propuesto proporciona una panorama de sus vías de influencia y productos.

Los think tanks pueden mejorar sus oportunidades de influencia en la medida que los productos de su experticia sean utilizados de manera directamente relevante a los propósitos para los cuáles han sido diseñados (Campbell, 1998: 154). De este modo, los centros poseen variaciones no sólo a nivel de sus orientaciones políticas e ideológicas, sino además respecto a la función preponderante que desempeñan. Ejemplo de ello son sus prácticas a nivel de la comunicación y difusión de las ideas. Un buen manejo de las estrategias comunicacionales puede ayudar a acortar las brechas entre la investigación desarrollada en los centros y la toma de decisiones en políticas (Bellettini, 2007). En efecto, el desarrollo de capacidades comunicativas y el mantenimiento de equipos periodísticos constituyen una de las innovaciones de los think tanks. Esto les ha permitido la exposición actualizada de sus actividades en la Web, su coordinación con medios de prensa, la publicitación de sus propuestas y la visibilización de sus expertos.

Mantener una imagen tal vez sea el principal valor comunicativo a la hora de presentar ideas en el debate público. Para ello se vuelve fundamental que los centros ideológicos logren guardar cierta autonomía de los grupos que los sostienen; de manera tal que su experticia sea evaluada en sus propios términos. Asimismo, los centros de cariz académico intentan cultivar una noción de imparcialidad frente a los asuntos que les competen. Para ello echan mano a una serie de recursos, ligados a una presentación rigurosa de los temas y a la apertura hacia diversas perspectivas. Las organizaciones partidarias, en cambio, por estar vinculadas a partidos políticos, tienen asegurado un mayor acceso a actores con poder de veto. El manejo de imagen pasa aquí menos por una noción de neutralidad que por la confianza que puedan brindar a los actores que apoyan. Un ejemplo de esto radica en la calidad y puntualidad de las asesorías ofrecidas a los parlamentarios de bancadas afines. Situación distinta a la que experimentan, por ejemplo, los centros de gestión, que deben mantener una imagen que muestre equilibrio entre la calidad de sus estudios, el respaldo en certificaciones y un perfil técnico.

Otro rasgo a destacar corresponde a la capacidad del centro para establecer una agenda de trabajo propia. Ya se ha mencionado que la planificación estratégica es transversal a estas organizaciones. Sin embargo, los think tanks se hallan sujetos a limitaciones de distinta índole. Los centros de gestión por lo general poseen una agenda fuertemente vinculada a los programas de las agencias ministeriales e instituciones encargadas de áreas de política. Los think tanks partidarios, por su parte, ven comprometida su agenda a las líneas programáticas de partidos y a actividades de formación de capital político. Los centros de apoyo y académicos en general logran construir agendas de trabajo de mediano plazo, aunque ciertamente condicionadas por la coyuntura. 
Ahora bien, no cualquier producto es óptimo para toda ocasión. La experticia como medio simbólico no posee un valor intrínseco, sino que es actualizado según su eficacia. Los productos ofrecidos deben poder hacer sentido entre los participantes de una controversia, y por lo tanto se someten a escrutinio en cada ocasión (Limoges, 1993). Por este motivo, los think tanks se han abocado a la elaboración de determinado tipo de recursos según sus propósitos y capacidades. Lo que se denomina gestión de la investigación pasa a jugar un rol fundamental (Braun et. al., 2007).

Los criterios de selección temática, procedimientos investigativos y características de los productos guardan diferencias entre los centros. Algunas de las publicaciones más populares de los think tanks pueden asociarse a las categorías que Rich (2004) ha utilizado en su análisis del ámbito norteamericano. Así, se observa un grueso de productos elaborados con fines preventivos o de interés cognitivo frente a temas, y otros que contienen connotaciones mayormente normativas sobre políticas específicas. Cada uno de ellos aporta información relevante respecto a la cualidad de la experticia gestionada en las organizaciones (Fig. 2).

\section{Figura 2. Esquema productos en think tanks}

\begin{tabular}{|c|c|c|c|}
\hline Centros & Productos & Énfasis & Interés \\
\hline De apoyo & $\begin{array}{l}\text { Propuestas, comentarios, } \\
\text { opiniones, difusión }\end{array}$ & $\begin{array}{l}\text { Estudios jurídicos, informes } \\
\text { económicos, columnas de } \\
\text { opinión }\end{array}$ & $\begin{array}{l}\text { Agenda de gobierno, } \\
\text { parlamento y grupos de } \\
\text { interés }\end{array}$ \\
\hline Partidarios & $\begin{array}{l}\text { Formación, intervención, } \\
\text { comentarios, propuestas, } \\
\text { opiniones }\end{array}$ & $\begin{array}{l}\text { Actividades de formación, } \\
\text { coyuntura política, estudios } \\
\text { jurídicos, columnas de opinión }\end{array}$ & $\begin{array}{l}\text { Agenda de partidos } \\
\text { políticos, de gobierno y } \\
\text { parlamento }\end{array}$ \\
\hline De gestión & $\begin{array}{l}\text { Propuestas técnicas, } \\
\text { estimaciones especificas, } \\
\text { estudios acotados }\end{array}$ & Informes técnicos & $\begin{array}{l}\text { Agenda de ministerios, } \\
\text { instituciones públicas y } \\
\text { parlamento }\end{array}$ \\
\hline Académicos & $\begin{array}{l}\text { Estudios generales sobre } \\
\text { asuntosy opciones, } \\
\text { propuestas temáticas, } \\
\text { actividades de difusión }\end{array}$ & $\begin{array}{l}\text { Investigaciones en } \\
\text { profundidad }\end{array}$ & $\begin{array}{l}\text { Agenda propia, agenda } \\
\text { de gobierno y } \\
\text { parlamento }\end{array}$ \\
\hline
\end{tabular}

Fuente: Elaboración propia

En las líneas de productos que exhiben los centros académicos puede observarse la edición de revistas periódicas y libros con estudios detallados de determinadas áreas de política. Las investigaciones preventivas (priming research) gozan de cierta popularidad al proveer percepciones generales de cómo problemáticas sociales, políticas o económicas pueden ser conducidas por los formuladores de políticas. De este modo, su experticia constituye una fuente de ideas para el debate, popularizando tópicos que en el corto plazo no siempre son factibles de re- 
solución desde el punto de vista técnico y político. A través de la visualización de problemas los documentos buscan concitar la atención de autoridades; de modo que sus esfuerzos incluso preceden la incorporación de asuntos a la agenda. Los think tanks se valen así de símbolos de autonomía y neutralidad, y sobresalen de otras organizaciones partidistas.

En esta línea de trabajos se identifican productos de centros académicos y de apoyo, entre los que destacan Estudios Públicos del CEP, Colección de Libros de CIEPLAN, Revista Proposiciones de SUR, y Estudios Sociales de CPU. Las colecciones son utilizadas para catapultar asuntos a la opinión pública y llamar la atención de las autoridades responsables.

Otra vertiente de productos se vincula mayormente a centros de apoyo. Está representada por lo que se denomina investigaciones sobre asuntos y opciones (issues and options research), y se definen por operar sintetizando dimensiones de problemas, para lo cual identifican rangos de alternativas plausibles. Ofrecen un mayor contenido normativo, en la medida que sus propuestas específicas suelen plantear justificaciones sobre opciones políticas preexistentes. Caracterizados por una menor extensión que los anteriores y por poseer un lenguaje simplificado, destacan aquí documentos como Serie en Foco de Expansiva, Temas Públicos del CEP, Revistas de la Fundación Paz Ciudadana, Asuntos Públicos del CED, Estudios y Propuestas de CIEPLAN, y Propuestas Públicas de Chile 21.

Think tanks partidarios y centros de apoyo elaboran además investigaciones de empuje (prodding research), que son aprovechadas para alentar a los formuladores de política en ciertas direcciones. Bajo la forma de columnas de opinión, reportes breves, apuntes y minutas, ofrecen recursos para respaldar u oponerse a determinados temas de la coyuntura, especialmente relativos a reformas a la legislación. Estudios con estas connotaciones, son Ideas y Propuestas de la Fundación Jaime Guzmán, Temas Públicos de Libertad y Desarrollo, Ensayos y Comentarios de CIEPLAN y la Colección Ideas de Chile 21.

Los centros de gestión, por su parte, han implementado criterios de especialización temática en sus productos. Estos a menudo fungen como evaluaciones a propuestas y programas de gobierno, aportando tanto en el monitoreo de las tareas como en el diseño de alternativas. Documentos como Conceptos de Fundación Paz Ciudadana, Informe Técnico Urbano de Fundación Jaime Guzmán y Serie Estudios de Libertar y Desarrollo, poseen estos atributos. 
Figura 3. Principales productos de think tanks en Chile

\begin{tabular}{|c|c|c|}
\hline \multirow{2}{*}{$\begin{array}{l}\text { Propósito de la } \\
\text { Investigación }\end{array}$} & \multicolumn{2}{|c|}{ Ámbito de Aplicación de la Investigación } \\
\hline & General & Especifico \\
\hline Prevenir & $\begin{array}{l}\text { Estudios Públicos(CEP) } \\
\text { Libros (CIEPLAN) } \\
\text { Proposiciones (SUR) } \\
\text { Estudios Sociales(CPU) }\end{array}$ & $\begin{array}{l}\text { Serie en Foco (Expansiva) } \\
\text { Temas Públicos (CEP) } \\
\text { Revistas (FPC) } \\
\text { Asuntos Públicos (CED) } \\
\text { Estudios y Propuestas (CIEPLAN) } \\
\text { Propuestas Públicas (Chile 21) }\end{array}$ \\
\hline Empujar, azuzar & $\begin{array}{l}\text { Ideas y Propuestas(FJG) } \\
\text { Temas Públicos (LyD) } \\
\text { Ensayos y Comentarios (CIEPLAN) } \\
\text { Colección Ideas (Chile 21) }\end{array}$ & $\begin{array}{l}\text { Informe Técnico Urbano (FJG) } \\
\text { Conceptos (FPC) } \\
\text { Serie Estudios (LyD) }\end{array}$ \\
\hline
\end{tabular}

Fuente: Elaboración propia

Como se observa, ciertos think tanks han logrado maximizar sus oportunidades estableciendo líneas de trabajo con diferentes características y prioridades (Fig. 3). Esto sirve tanto para relativizar un esquema demasiado rígido de clasificación, como para enfatizar la idea de apertura cognitiva de estas instituciones; esto es, su capacidad de aprendizaje y sondeo del medio político en que actúan.

\section{Conclusiones}

La proliferación de centros de estudio privados o think tanks, y su asentamiento en cuanto actor con relativa participación en el proceso político, representa una tendencia consolidada en el país. Aunque lejos de la situación anglosajona, la experiencia chilena parece confirmar la influencia estable que han alcanzado estas organizaciones durante las últimas tres décadas. Esto se debe, entre otras causas, a la participación que tuvieron durante el proceso de transición democrática y su apoyo a la consolidación institucional de gobiernos posteriores.

El artículo ha puesto en relieve la situación de lo que puede denominarse la tercera generación think tanks en el país; destacando cómo estas organizaciones logran albergan actores heterogéneos desde el punto de vista ideológico y operativo. En efecto, los centros de estudio privados han ganado no sólo en diversidad ideológica sino también en la dimensión funcional: dando origen a think tanks con prioridades distintas, capaces de desarrollar estrategias diferenciadas, además de canales y recursos especializados para codificar su experticia e incrementar sus oportunidades de influencia. 
En breve, la investigación sugiere que la intervención de los think tanks en el proceso de política pública se explica en buena medida por la gestión de una determinada experticia, la que es cursada por vías y recursos particulares según las características e intereses de las organizaciones. Para ilustrar este mecanismo es revelador observar su participación en el establecimiento de agendas.

En centros de apoyo, los medios de influencia se hallan estrechamente asociados a una experticia que funge como dispositivo para la simplificación y orientación de la evidencia disponible, y su transmisión a audiencias amplias a través de medios de difusión -prensa escrita, radio, internet. Aquí, la exposición mediática, el prestigio institucional y la imagen del think tank poseen un valor crítico a la hora de legitimar ofertas de experticia, dando así impresión de autonomía e independencia en sus labores. A esto se añade la capacidad de vínculo con expertos y actores políticos de cierto prestigio, como forma de conseguir acceso e influir sobre la toma de decisiones.

Como organizaciones derivadas de las anteriores, los centros partidarios han encontrado en el país instituciones y partidos políticos estables a los cuáles asesorar en materias de interés. La experticia desarrollada permite seleccionar y sintetizar materias complejas, reelaborando los asuntos de política con objeto de alimentar el debate interno de partidos, así como brindar asesoría a representantes parlamentarios y de gobierno afines. Los think tanks dotan a grupos políticos de la reflexividad requerida para el posicionamiento en controversias donde, la mayor parte de las veces, autoridades y actores tradicionales declaran ignorancia. A diferencia de los anteriores, sus prestaciones tienden a circular en redes menos publicitadas, facilitadas por el acceso a jugadores con poder de veto. No obstante, su rasgo distintivo refiere a un uso eminentemente "interventor" de la experticia, escenificado en instancias diseñadas para la formación y capitalización política de sus audiencias.

Los centros de gestión, por su parte, generan una experticia centrada en la producción de informes técnicos y evaluaciones de intervenciones de política. Las características de esta labor impelen una especialización temática en áreas acotadas de la política pública, por lo que su impacto tiende a limitarse a campos exclusivos. Como contraparte, el nivel de detalle de las sugerencias que prestan suele concitar una importante atención en la elaboración de alternativas para agendas sectoriales.

Centros académicos, finalmente, han destacado por desenvolverse en medios que convocan una cultura académica, ligada a la investigación aplicada en ciencias económicas y sociales, con directrices ideológicas débiles o difusas. De este modo, su experticia se canaliza vía la instalación de agendas de trabajo propias, la formación de equipos de investigación estables y una legitimidad relativamente favorable a sus productos entre la opinión pública. 
Como puede constatarse, existe una diferenciación a nivel de la gestión de experticia de los think tanks y los recursos que utilizan para operacionalizar sus pretensiones de influencia. Esto no debe tomarse, empero, como un esquema rígido de comportamiento. Si bien manifiestan prioridades, las organizaciones pueden manejar más de un único recurso para alcanzar sus objetivos. Con esto, los think tanks cultivan capacidades para desplegar su experticia en múltiples ámbitos del ciclo de políticas, obteniendo en sus intervenciones distintos grados de efectividad.

Finalmente, es necesario reflexionar sobre sesgos y definiciones que imprime el saber experto en la toma de decisiones sobre políticas, así como por el impacto que tienen los think tanks en la vida democrática. En términos amplios, el debate se ha enfocado en cómo y en qué medida la gestión de determinada experticia puede llegar a constituir un insumo valioso para la formación de políticas de calidad. En torno a este tema han surgido al menos dos problemas. El primero de ellos dice relación con las "premisas de participación” involucradas en la toma de decisiones. En efecto, los procesos deliberativos en una democracia suponen la apertura de ámbitos decisionales a la ciudadanía, tanto de manera directa como a través de representantes electos. En la actualidad, la gestión de experticia por parte de think tanks particulares puede afectar las premisas con las cuáles se discrimina la participación política, generando un sesgo en la diversidad de visiones que debiese estar plasmada en la definición de los asuntos públicos. En un nivel discursivo ello ha contribuido a generar una importante carga de valoración negativa sobre el saber experto, especialmente por parte de movimientos sociales y políticos que aspiran a ampliar los espacios de participación y deliberación.

El segundo foco problemático estriba en las "premisas de legitimidad”. El cuestionamiento surge cuando se observa que el saber experto es colocado como un elemento para justificar técnicamente decisiones vinculantes de carácter eminentemente político. Es parte de la crítica que versa sobre los programas tecnocráticos, donde la contribución experta propende a oscurecer y ocultar voluntades normativas e ideológicas. Para esto el medio de la experticia ha parasitado los códigos del conocimiento científico, presentándose a sí mismo en forma de argumentos verdaderos e indubitables. Las consecuencias de estos fenómenos relevan preguntas fundamentales sobre las normas y valores que orientan la definición de los asuntos públicos en democracia. 
Polis, Revista de la Universidad Bolivariana, Volumen 11, $N^{\circ}$ 32, 2012

\section{Notas}

${ }^{*}$ El artículo fue presentado como ponencia en el $6^{\circ}$ Congreso Chileno de Sociología y Encuentro PreAlAS Chile 2011. Sociología y Sociedad en Chile: Escenarios y Diálogos Contemporáneos, Universidad de Playa Ancha, Universidad de Valparaíso, Sociored Chile, Santiago, 13-15 de Abril de 2011. El autor agradece a los asistentes a la exposición, así como a Francisco Godoy y Marcelo Arnold Cathalifaud por sus inestimables observaciones durante el proceso de investigación.

${ }^{1}$ Para una explicación metodológica del esquema, y la discusión entre “criterios ideológicos” y “criterios operativos” de clasificación, consultar Pinilla y Godoy (2010). 


\section{Bibliografía}

Abelson, Donald (2009), Do Think Tanks Matter? Assessing the Impact of Public Policy Institutes. $2^{\text {nd }}$ ed. McGill-Queen's University Press, Québec.

Abelson, Donald and Carberry, Christine (1998), "Following Suite or Falling Behind? A Comparative Analysis of Think Tanks in Canada and the United States". Canadian Journal of Political Sciences, 31(3), 525555.

Aguilera, Carolina (2009), "Las Comisiones Asesoras Presidenciales del Gobierno de Michelle Bachelet”. Documento de Trabajo del Programa de Gobernabilidad. FLACSO, Santiago.

Ídem (2007), "Participación Ciudadana en el Gobierno de Bachelet: Consejos Asesores Presidenciales”. América Latina Hoy, 46: 119-143.

Baier, Gerald and Bakvis, Herman (2007), “Think tanks y partidos políticos en Canadá: ¿competidores o colaboradores?”. En: Adolfo Garcé y Gerardo Uña, Think tanks y políticas públicas en Latinoamérica: dinámicas globales y realidades regionales. Prometeo Libros, 51-67, Buenos Aires.

Bellettini, Orazio (2007), "El papel de los centros de política pública en las reformas públicas implementadas en América Latina”. En, Garcé, Adolfo y Uña, Gerardo (comps.). Think tanks y políticas públicas en Latinoamérica: dinámicas globales y realidades regionales. Prometeo Libros, 111-137, Buenos Aires.

Braun, Miguel; Chudnovsky, Mariana; Dacuté, Nicolás; Weyrauch, Vanesa (2007), "Lejos de 'Thinktanklandia': los institutos de investigación de políticas en los países en desarrollo”. En: Adolfo Garcé y Gerardo Uña, Think tanks y políticas públicas en Latinoamérica: dinámicas globales y realidades regionales. Prometeo Libros, 69-110, Buenos Aires.

Brunner, José Joaquín (1985), “La participación de los Centros Académicos Privados”. Estudios Públicos, 19, 1-12.

Campbell, John L (1998), "Institutional Analysis and the Role of Ideas in Political Economy”. Theory and Society, 27(3), 377-409.

Centeno, Miguel (1993), "The new Leviathan: the dynamics and limits of technocracy”. Theory and Society, 22, 307-335.

Centeno, Miguel and Silva, Patricio (1998), "The Politics of Expertise in Latin America: Introduction”. En: Miguel Centeno and Patricio Silva (ed.), The Politics of Expertise in Latin America. St. Martin's Press, Inc., 1-11, New York. 
Duflo, Esther and Takavarasha, Kudzai (2010), "Social science and policy design”. En, UNESCO and ISSC. World Social Science Report. Unesco, 330-332, París.

European Commission (2008), "Scientific evidence for policy-making”. Directorate-General for Research Socio-economic Sciences and Humanities. Luxembourg, Office for Official Publications of the European Communities.

Fischer, Frank (2009), Democracy and Expertise. Reorienting Policy Inquiry. Oxford University Press, New York.

Gárate, Manuel (2008), “Think Tanks y Centros de Estudio. Los nuevos mecanismos de influencia política en el Chile post-autoritario”. En: Maite de Cea, Paola Díaz y Géraldine Kerneur. Chile: ¿De país modelado a país modelo? Una mirada sobre la política, lo social y la economía. LOM Ediciones, 67-85, Santiago.

Ídem (2010), “CIEPLAN entre dictadura y democracia: orígenes y alcances de un Think Tank de economistas”. Ponencia presentada en el Congreso "Produciendo Lo Social: Una Mirada Reflexiva a las Ciencias Sociales en Chile y América Latina”, Facultad de Ciencias Sociales e Historia, Universidad Diego Portales, 25 de Octubre de 2010, Santiago de Chile.

Howlett, Michael \& Ramesh, M (2003), Studying public policy: policy cyles and policy subsystems. $2^{\text {nd }}$ ed., Oxford University Press, Canada.

Joignant, Alfredo (2004), Profesionalización de la política y política de calidad: Elementos de análisis. Colección Ideas, 4(43).

Kingdon, John (1995), Agendas, Alternatives, and Public Policies. $2^{\text {nd }}$ ed., Harper Collins College Publishers, New York.

Limoges, Camille (1993), "Expert knowledge and decision-making in controversy contexts”. Public Understanding of Science, 2, 417-423.

McGann, James dir. (2011), “The Global ‘Go-To Think Tanks’ 2010. The Leading Public Policy Research Organizations in the World”. Think Tanks and Civil Socities Program, University of Pennsylvania.

Ídem (2008), “The Global 'Go-To Think Tanks. The Leading Public Policy Research Organizations in the World”. Think Tanks and Civil Socities Program, University of Pennsylvania.

Markoff, John and Montecinos, Verónica (1994), “El irresistible ascenso de los economistas”. Desarrollo Económico, 34(133): 3-29.

Pinilla, Juan Pablo y Godoy, Francisco (2010), “Las Formas 
Organizacionales del Conocimiento Experto. Think Tanks y Política en el Chile Actual”. Congreso Produciendo Lo Social: Una Mirada Reflexiva a las Ciencias Sociales en Chile y América Latina, Facultad de Ciencias Sociales e Historia, Universidad Diego Portales, 25 de Octubre de 2010, Santiago de Chile,.

Puryear, Jeffrey (1994), Thinking politics: intellectuals and democracy in Chile, 1973-1988. The Johns Hopkins University Press, Baltimore.

Rich, Andrew (2004), Think Tanks, Public Policy, and the Politics of Expertise. Cambridge University Press, New York.

Silva, Patricio. (1991), “Technocrats and politics in Chile: From the Chicago Boys to the CIEPLAN Monks”. Journal of Latin American Studies, 23(2), 385-410.

Ídem (2006), “Los tecnócratas y la política en Chile: Pasado y presente”. Revista de Ciencia Política, 26(2), 175-190.

Ídem (2007), “Estilos políticos y orientación tecnocrática bajo los gobiernos de Lagos y Bachelet”. Revista de Sociología, 21, 79-105.

UNESCO and ISSC (2010), "The political use and abuse of social sciences”. En: World Social Science Report. Unesco, 318, París.

Uña, Gerardo (2007), “Think tanks en Argentina: sobreviviendo a la tensión entre la participación y la permanencia”. En: Adolfo Garcé y Gerardo Uña, Think tanks y políticas públicas en Latinoamérica: dinámicas globales y realidades regionales. Prometeo Libros, 177-220, Buenos Aires.

Wedel, Janine; Shore, Cris; Feldman; Gregory and Lathrop, Stacy (2005), "Toward an Anthropology of Public Policy". The ANNALS of the American Academy of Political and Social Science, 600, 30-51.

Willke, Helmut. (2006), "La transformación de la democracia como modelo de orientación de las sociedades complejas”. Estudios Públicos, 102, 179-201.

Ídem (2007), Smart Governance. Governing the Global Knowledge Society. University of Chicago Press, Chicago.

\section{Entrevistas}

Alfredo Joignant, Corporación Expansiva. Harald Beyer, Centro de Estudios Públicos. Jaime Bellolio, Fundación Jaime Guzmán. Manuel Riesco, CENDA. 
Polis, Revista de la Universidad Bolivariana, Volumen 11, $N^{\circ}$ 32, 2012

Patricio Tudela, Fundación Paz Ciudadana.

Rafael Urriola, Fundación Chile 21.

Tomás Flores, Libertad y Desarrollo.

Recibido: 26.08.2011

Aceptado: 25.07.2012 